\title{
Multicentric chondrosarcoma involving the appendicular skeleton: a case report and literature review
}

\author{
V. Aran $\mathrm{PhD}^{*}{ }^{*}$ W. Meohas $\mathrm{MD}_{1}^{\dagger}$ A.C. de Sá Lopes $\mathrm{MD}_{,}^{\dagger}$ L. Maciel Cabral $\mathrm{MD}_{1}^{\ddagger}$ \\ A. Fortuna-Costa PhD, ${ }^{*}$ J.A. Matheus Guimarães MD PhD, ${ }^{*}$ and M.E. Leite Duarte MD PhD*
}

\begin{abstract}
Chondrosarcomas constitute the 3rd most common primary bone malignancy. These tumours grow slowly and rarely metastasize, usually having a good prognosis after surgery. Among patients registered and treated at the Brazilian National Institute of Traumatology and Orthopedics, an uncommon case of chondrosarcoma was identified in a 63-year-old man, who was diagnosed with multicentric chondrosarcoma of the appendicular skeleton. This example is atypical in the medical literature because multicentric tumours are different from metastatic events, and their frequency in chondrosarcoma is rare. This article therefore provides a rare case report alongside a review of additional cases in the medical literature.
\end{abstract}

Key Words Bone sarcomas, chondrosarcoma, multicentric tumours, appendicular skeleton

\section{INTRODUCTION}

Bone sarcomas constitute a heterogeneous group of rare malignant tumours, characterized by various degrees of mesenchymal differentiation. Sarcomas are classified based on parameters such as cell type, specific histologic characteristics, relation of the lesion to bone tissue, presence of pre-existing conditions, and other biologic characteristics. They constitute $0.2 \%$ of all adult malignancies and $5 \%$ of childhood and adolescent malignancies ${ }^{1}$.

Although bone sarcomas are diagnosed in all age groups, a relationship between age, location, and tumour type has been observed ${ }^{2}$. Of sarcomas affecting all skeletal bones, the distribution between male and female patients is $1: 0.7$. Some tumours show a predilection for the bones of the pelvis, axial skeleton, and long bones, but rarely the bones of the hands and feet ${ }^{3}$. With respect to tumour types, a large study conducted in the United Kingdom (9146 cases) distributed bone cancer types by morphology, with osteosarcoma being the most frequent primary bone sarcoma $(34.2 \%)$, followed by chondrosarcoma (27.2\%), Ewing sarcoma (19.3\%), and others (19.4\%) ${ }^{4}$. The incidence also varied with age, such that Ewing sarcoma was most common in patients 0-9 years of age, osteosarcoma in those 10-29 years of age, and chondrosarcoma in those 30-84 years of age ${ }^{4}$. Interestingly, the incidence of chondrosarcoma increased steadily with age. For 2020, the American Cancer Society (https://www.cancer.org/) estimates 3500 new diagnosed cases and 1660 deaths. The incidence varies, with approximately $40 \%$ of primary bone cancers in adults being chondrosarcomas; in children and adolescents, chondrosarcomas are less common $(\sim 6 \%)$.

\section{Chondrosarcoma}

Cartilage tumours present in various sizes and grades. They range from benign lesions such as enchondromas and osteochondromas, to malignant chondrosarcomas. The latter are part of a heterogeneous group that constitutes approximately $20 \%-25 \%$ of all primary malignant bone neoplasms ${ }^{5}$. Classical chondrosarcoma can be divided into grade I (low grade), II (intermediate grade), and III (high grade) tumours. Other histologic types include periosteal chondrosarcoma and clear, mesenchymal, and undifferentiated cells. In addition, chondrosarcomas can be further classified as medullary or peripheral, according to their position in relation to the bone marrow. Most are low-grade lesions originating from cartilaginous dysplastic processes ("secondary chondrosarcomas"). The mesenchymal subtype tends to behave malignantly and presents with a high propensity to bone metastasis and multicentricity. To distinguish benign cartilage tumours from low-grade

Correspondence to: Veronica Aran, Research Division, National Institute of Traumatology and Orthopaedics, 500 Avenida Brasil, São Cristóvão, Rio de Janeiro-RJ 
chondrosarcomas, a combination of radiographic techniques and histologic analyses is usually required.

Chondrosarcomas contain cells that produce chondroid matrix. Interestingly, because of endochondral ossification, they are commonly found in the bones that elongate, such as the proximal and distal femur, the proximal humerus, and ribs ${ }^{6}$. They usually affect people more than 40 years of age, and incidence rates increase with age. Although there is some evidence that ionizing radiation might play a causative role, the risk factors for chondrosarcoma are unknown ${ }^{7}$. Deregulation of the Hedgehog signalling pathway, which is involved in normal bone development, can contribute to benign lesions ${ }^{8}$. Those lesions can be the precursors to malignant chondrosarcomas, which are often resistant to conventional chemotherapy and radiotherapy ${ }^{8}$.

Most chondrosarcoma tumours grow slowly, and they rarely metastasize, making for an excellent prognosis after surgery ${ }^{9}$. Given their extracellular matrix, low percentage of dividing cells, and poor vascularity, they are relatively resistant to chemotherapy and radiotherapy, leaving surgical excision as the best treatment available for intermediate-grade to high-grade tumours. Few patients present with metastases or recurrences, and approximately $13 \%$ of recurrent chondrosarcomas are of a higher grade than the original tumour ${ }^{9}$.

\section{Chondrosarcoma and Multicentricity}

Multicentricity in malignancy is often difficult to distinguish from metastatic involvement. Multicentric tumours are uncommon, given that they develop at the same time in more than one organ or site, but without a clonal relation $^{10}$. Conventional chondrosarcomas rarely present with metastases, and it is rare to observe multicentric behaviour in malignant cartilage tumours ${ }^{11}$. Other than patients with mesenchymal chondrosarcomas, the greatest proportion of individuals with chondrosarcoma do not show multicentricity, including patients with multiple exostoses or with enchondromatosis ${ }^{12}$.

Classically, multicentricity has been the accepted explanation for multiple or metachronous skeletal malignancies when they appear in the absence of visceral involvement ${ }^{13}$. Synchronous tumours present simultaneously at the time of diagnosis or at the time of surgery (contiguous lesions being ruled out); metachronous tumours are found during follow-up of patients already having undergone surgical resection, evidently excluding the possibility of an undiagnosed lesion at the time of diagnosis (that is, when it would be synchronous) or recurrence of the resected tumour ${ }^{14}$.

After careful evaluation of the patient's clinical data, the case described in this report was considered to represent a rare case of multicentricity. This case study was approved by the Human Ethics Committee of the Brazilian National Institute of Traumatology and Orthopedics (protocol no. 16772619.1.0000.5273).

\section{CASE DESCRIPTION}

A 63-year-old man was referred from a small local hospital to the Brazilian National Institute of Traumatology and Orthopedics, one of the leading hospitals in Latin America, specialized in the orthopedics and traumatology fields. He was admitted in 2018, reporting pain in the right forearm, which he said had been present for 5 months, with progressive worsening. In the outpatient clinic, the patient reported that, previously, in 2006, he had had a tumour mass in the popliteal region, which was resected and diagnosed as benign. Years later, in 2010, the patient presented with recurrence, and a new resection was performed after a diagnosis of soft-tissue myxoid chondrosarcoma in the right popliteal fossa, which led to transfemoral amputation, with no signs of metastasis.

Once the patient was admitted to our institution in 2018, he underwent both radiographic and magnetic resonance imaging examinations [Figure $1(\mathrm{~A}, \mathrm{~B})$ ], which revealed a soft-tissue mass in the central region of the right forearm. He next underwent incisional biopsy of the lesion, and after careful morphologic evaluation and histologic analysis, the diagnosis of high-grade myxoid chondrosarcoma was made (Figure 2, showing a tumour with chondrocytes irregularly dispersed). Surgical resection with clear margins was subsequently performed [Figure 1(C,D)].

However, in 2019, the patient experienced a new local recurrence, with an indication for transhumeral amputation. No pulmonary metastasis was observed in chest computed tomography imaging (Figure 3), and the patient did not receive adjuvant therapy, given that the two malignant findings were considered to be unrelated lesions. The morphologic and histopathologic characteristics of
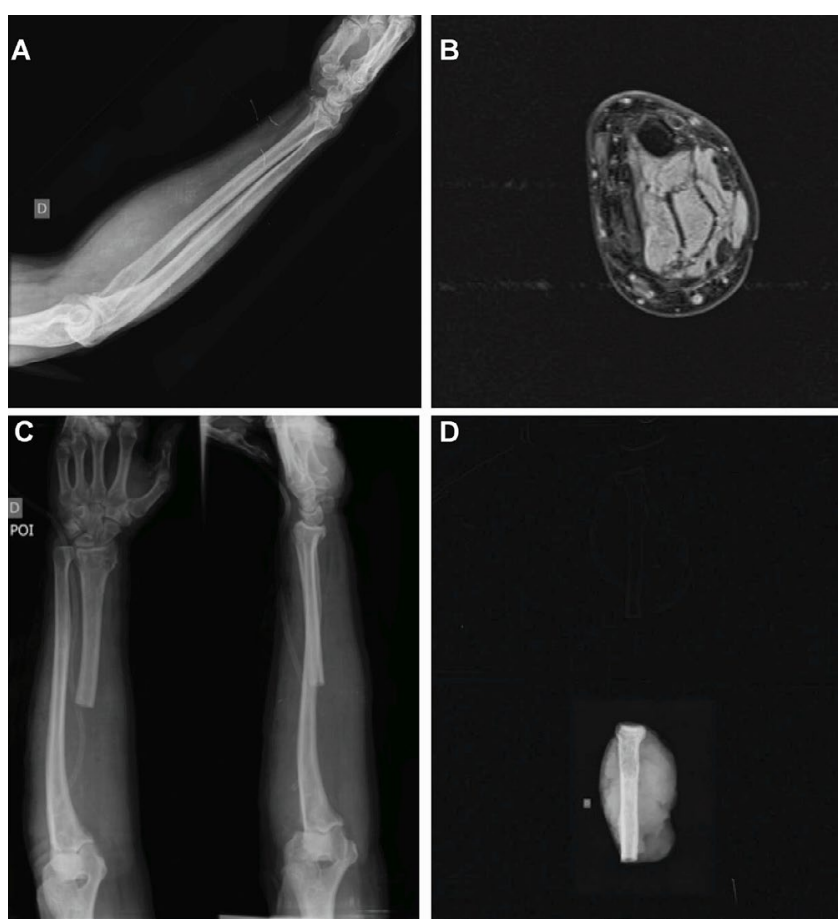

FIGURE 1 Radiographic and magnetic resonance images. (A) Preoperative lateral radiograph of forearm showing soft-tissue radiopaque mass in the central region of the limb. (B) T2-Weighted magnetic resonance image showing the axial section of forearm containing the tumour mass. (C) Postoperative image showing anteroposterior and resection profile. (D) Radiograph of the resected surgical specimen. 

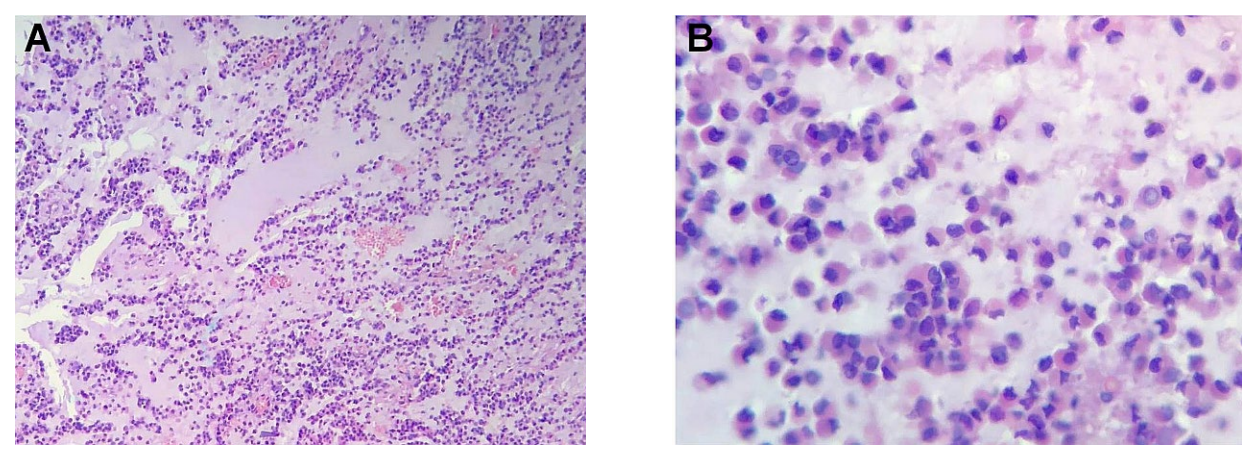

FIGURE 2 Histopathology image of the resected high-grade chondrosarcoma under hematoxylin-eosin staining (A) at 100× and (B) 400× original magnification. The images show disorganized chondrocytes and chondral matrix.
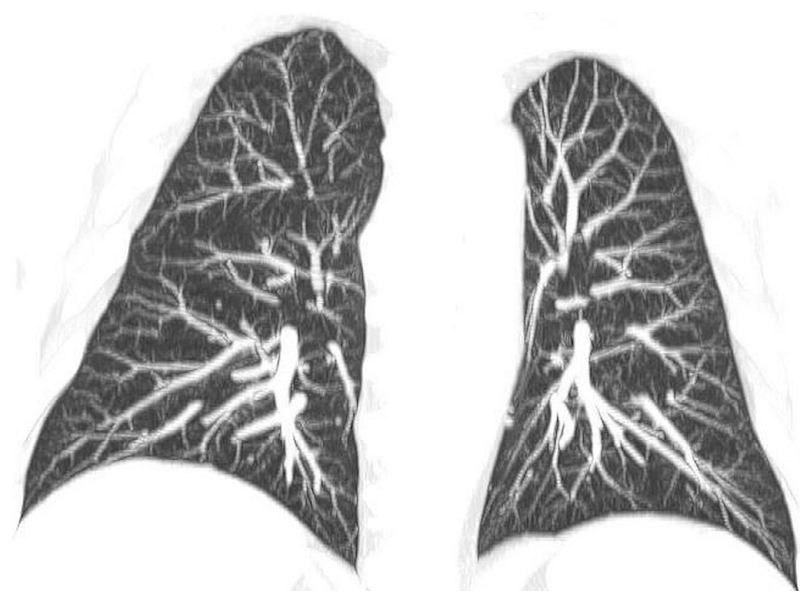

FIGURE 3 Chest computed tomography (CT) of the lungs showing the absence of pulmonary metastasis.

the tumours led us to the conclusion that this was a multicentric myxoid chondrosarcoma—an infrequent finding.

\section{DISCUSSION}

Multicentric chondrosarcomas are rare and difficult to differentiate from metastatic disease. Only a few reports have been published in the literature.

A 1996 study described 8 patients with multicentric chondrosarcomas ${ }^{12}$. Of those 8 patients, 5 had monomeric chondrosarcomas, 3 had disseminated chondrosarcomas, 3 had synchronous involvement, 5 had metachronous involvement, and 1 patient had Ollier disease. Each of the patients with synchronous chondrosarcomas had single-limb involvement of the lower limbs. In all 5 patients with metachronous chondrosarcomas, the second tumour involved a different bone. However, only in 1 patient were the metachronous chondrosarcomas limited to the lower extremity ${ }^{12}$.

Another study described a case in which two separate sites of chondrosarcoma were found and treated as multicentric chondrosarcomas in a patient with Ollier disease ${ }^{15}$. Our patient did not have Ollier disease.

A more recent case of multicentric chondrosarcoma in a 40-year-old female patient described two non-contiguous chondrosarcomas, one originating in the left pelvic region and one originating in the right scalp, with no evidence of visceral or pulmonary metastases ${ }^{11}$. Our patient similarly did not show pulmonary metastasis.

Alongside our report, these few cases are important to the medical literature, given that interpreting multicentric tumours as metastatic tumours could lead to an erroneous diagnosis and consequently an inappropriate treatment choice.

\section{SUMMARY}

Our patient with multicentric myxoid chondrosarcoma underwent both transfemoral and transhumeral amputation. Multicentric chondrosarcoma is an uncommon finding because metastatic processes are more frequent than multicentric processes. The scarcity of such case reports in the medical literature confirms the rarity of multicentric chondrosarcomas and thus the importance of describing the condition when it appears. The present report might work to draw attention to the appropriate investigation of patients with similar characteristics so as to distinguish multicentric tumours from metastatic events.

\section{ACKNOWLEDGMENTS}

We thank the patient for granting permission to present this case study.

\section{CONFLICT OF INTEREST DISCLOSURES}

We have read and understood Current Oncology's policy on disclosing conflicts of interest, and we declare that we have none.

\section{AUTHOR AFFILIATIONS}

*Research Division, ${ }^{\dagger}$ Department of Oncology, and ${ }^{\ddagger}$ Department of Pathology, National Institute of Traumatology and Orthopaedics, Rio de Janeiro, Brazil.

\section{REFERENCES}

1. Fletcher CDM, Bridge JA, Hogendoorn PCW, Mertens F. WHO Classification of Tumours of Soft Tissue and Bone. 4th ed. Lyon, France: IARC Press; 2013.

2. Harting MT, Lally KP, Andrassy RJ, et al. Age as a prognostic factor for patients with osteosarcoma: an analysis of 438 patients. J Cancer Res Clin Oncol 2010;136:561-70.

3. Dorfman HD, Czerniak B. Bone cancers. Cancer 1995; 75(suppl):203-10. 
4. Arora RS, Alston RD, Eden TOB, Geraci M, Birch JM. The contrasting age-incidence patterns of bone tumours in teenagers and young adults: implications for aetiology. Int $J$ Cancer 2012;131:1678-85.

5. Murphey MD, Walker EA, Wilson AJ, Kransdorf MJ, Temple HT, Gannon FH. From the archives of the AFIP: imaging of primary chondrosarcoma: radiologic-pathologic correlation. Radiographics 2003;231245-78.

6. Dai X, Ma W, He X, Jha RK. Review of therapeutic strategies for osteosarcoma, chondrosarcoma, and Ewing's sarcoma. Med Sci Monit 2011;17:RA177-90.

7. Valery PC, Laversanne M, Bray F. Bone cancer incidence by morphological subtype: a global assessment. Cancer Causes Control 2015;26:1127-39.

8. Bovée JV, Hogendoorn PC, Wunder JS, Alman BA. Cartilage tumours and bone development: molecular pathology and possible therapeutic targets. Nat Rev Cancer 2010;10:481-8.

9. Gelderblom H, Hogendoorn PC, Dijkstra SD, et al. The clinical approach towards chondrosarcoma. Oncologist 2008;13:320-9.
10. de Werra C, Donzelli I, Perone M, Di Micco R, Orabona G. Multifocal and multicentric tumors. In: Renda A. Multiple Primary Malignancies. New York, NY: Springer Publishing Company; 2009.

11. Choong C, Chan H, Azuhairy A, Anwar Hau M, Zulkiflee O. A rare presentation of metachronous multicentric pelvic and extracranial chondrosarcoma: a case report. Malays Orthop J 2014;8:55-8.

12. Damron TA, Sim FH, Unni KK. Multicentric chondrosarcomas. Clin Orthop Relat Res 1996;:211-19.

13. Fitzgerald RH Jr, Dahlin DC, Sim FH. Multiple metachronous osteogenic sarcoma. Report of twelve cases with two longterm survivors. J Bone Joint Surg Am 1973;55:595-605.

14. Da Silva Andrade SM, Pereira FL. Synchronic colorectal cancer-case report and literature review [Portuguese]. Rev Bras Coloproctol 2007;27:69-79.

15. Lucas D, Tupler R, Enneking WF. Multicentric chondrosarcomas associated with Ollier's disease. Review and case report. J Fla Med Assoc 1990;77:24-8. 